\title{
Avaliação do uso de agente compatibilizante no comportamento mecânico dos compósitos PEBD reforçados com Fibras de Coco Verde
}

\section{Evaluation of the use of compatibilizer in the mechanical behavior of LDPE reinforced with green coconut fibers composites}

Artigo

Original

Taysa Ávila Oliveira ${ }^{1}$

Alison Teixeira ${ }^{1}$

Original

Daniella Regina Mulinari²

Paper

Shane Aparecida Soares Goulart ${ }^{2}$

\begin{abstract}
Palavras-chave:
Compósitos poliméricos

\section{Resumo}

Fibras de Coco Verde

Propriedades mecânicas

Nesta pesquisa, amostras de polietileno de baixa densidade (PEBD) e compósitos de fibra de coco verde reforçados em polietileno de baixa densidade, não compatibilizados e compatibilizados, foram avaliadas quanto às propriedades mecânicas (tração). As superfícies fraturadas dos compósitos ensaiados foram analisadas. Os resultados obtidos revelaram que os compósitos de fibras de coco reforçados com polietileno de baixa Agent Compatibilizante densidade compatibilizados apresentaram melhores propriedades mecânicas quando comparados aos compósitos não compatibilizados.
\end{abstract}

Abstract

In this research samples of low density polyethylene and green coconut fibers reinforced by low density polyethylene composites, compatibilizer and non compatibilizer, were evaluated concerning their mechanical properties (tensile). The fractured surface of the tested composites were analyzed. The results showed that the green coconut fibers reinforced by low density plyethylene composites compatibilizer presented better mechanical properties when compared to the non compatibilizer.
Key words:

Polymerics composites

Green coconut fibers

Mechanical properties

Compatibilizer 


\section{Introdução}

Nos últimos anos, houve um aumento no desenvolvimento de novas tecnologias e tecnologias que possibilitem a utilização de produtos com menor impacto ambiental. A sustentabilidade ambiental é um objetivo a ser atingido, mas nem tudo que apresenta algumas melhorias em temas ambientais pode ser considerado realmente sustentável ${ }^{1}$.

Nesse contexto, os compósitos poliméricos reforçados com fibras naturais têm recebido especial atenção devido às vantagens das fibras naturais quando comparadas às fibras sintéticas, tais como; são de provenientes de fontes renováveis, possuem baixa densidade e baixo custo, não são tóxicas, podem ser incineradas, são biodegradáveis e, principalmente, por serem consideradas sustentáveis ${ }^{2,3}$. Outro fator importante que favorece o emprego de fibras naturais como insumo renovável é a crescente perspectiva de economia de energia por meio da redução de peso dos componentes, bem como os aspectos ligados à recuperação das matérias-primas e ao reaproveitamento dos materiais no final do ciclo de vida do produto ${ }^{4}$.

Além disso, os resíduos dessas fibras são gerados em grande quantidade e a proposta de utilização, como por exemplo, o coco verde é muito interessante. A fibra proveniente do coco verde possui relativa vantagem com relação à abundância e custo, já que é o Brasil é um grande consumidor de coco verde.

No entanto, a compatibilidade entre um material lignocelulósico e uma matriz polimérica possui um papel determinante nas propriedades do compósito final, pois, é através da interface que ocorre a transferência de carga da matriz para a fibra.

Quando há incompatibilidade, a interface é a região mais fraca do material, local onde ocorre a falha, que acaba comprometendo o reforço do compósito, devido à transferência ineficiente de esforços na interface fibra/ matriz ${ }^{5}$. Muitos trabalhos indicam que a modificação superficial das fibras naturais antes de ser incorporada como reforço em matrizes poliméricas reduz a diferença de polaridade e melhora a compatibilidade entre fibra e matriz ${ }^{6-}$ 12. Outros trabalhos também indicam adicionar agentes compatibilizantes ${ }^{13}$. Os agentes com- patibilizantes mais utilizados são o polipropileno graftizado com anidrido maleico (PP-gMA) e o polietileno graftizado com anidrido maleico (PE-g-MA). Além da incorporação do agente compatibilizante, um processo de mistura adequado deve ser conduzido e o tamanho das fibras, controlado.

Nos estudos de propriedades mecânicas, entre os quais estão os ensaios de tração, impacto e flexão, tem-se observado que a incorporação de agentes compatibilizantes é imprescindível para a obtenção de resistências à tração e flexão superiores àquelas obtidas nos polímeros puros.

Portanto, o objetivo deste trabalho foi avaliar o uso do agente compatibilizante no compósito polimérico reforçado com fibras de coco verde, agregando-se valor à matériaprima pelo uso mais nobre e contribuindo com a criação de um material que possui propriedades mecânicas e físico-químicas satisfatórias para o uso industrial.

O compósito polimérico reforçado com fibras naturais é um material interessante, por ser altamente eficiente e, principalmente, por não poluir o ambiente de trabalho, que é um dos grandes problemas para quem trabalha com polímeros. Além disso, a ISO 14000 dá a esses compósitos a chance de serem considerados não somente como uma alternativa isolada, mas também uma estratégia para reduzir problemas ambientais.

\section{Materiais e Metódos}

\subsection{Obtenção das Fibras de Coco Verde}

As fibras de coco foram gentilmente fornecidas pelo Projeto Coco Verde. Primeiramente as fibras foram cortadas em tamanhos de aproximadamente $5 \mathrm{~cm}$ e moídas em um moinho de facas. Em seguida foram peneiradas em uma peneira de 25 mesh. A morfologia das fibras de coco verde foi analisada pela técnica de microscopia eletrônica de varredura (MEV).

\subsection{Preparação dos Compósitos}

Os compósitos foram obtidos em um homogeneizador de plásticos de laboratório 
da MH Equipamentos (modelo MH-50H), disponível na Divisão de Materiais (AMR) do Comando-Geral de Tecnologia Aeroespacial (CTA). A mistura entre as fibras de coco, o polímero e o agente compatibilizante ocorreram dentro da cápsula bipartida, com arrefecimento de água. As palhetas de homogeneização giraram com, aproximadamente, $2600 \mathrm{rpm}$ na primeira velocidade e $5250 \mathrm{rpm}$ na segunda, tornando o processo de homogeneização rápido. A primeira velocidade serviu para tirar o motor e o eixo do ponto de inércia e a segunda para misturar os materiais. E foi nesse momento que o equipamento foi desligado para que não ocorresse a degradação do material.

Para a obtenção dos compósitos, primeiramente as fibras de coco verde foram secas em estufa a $50{ }^{\circ} \mathrm{C}$ por $3 \mathrm{~h}$. O PEBD, fornecido pela CROMASTER, também foi seco á mesma temperatura, porém, por $1 \mathrm{~h}$. Posteriormente, os materiais foram pesados nas proporções de $5(\mathrm{~m} / \mathrm{m})$ de fibras. Foram preparados cerca de
400 g de cada material compósito em bateladas de $50 \mathrm{~g}$ no homogeneizador de plásticos. Após a mistura, o material fundido passou entre rolos de aços inox (calandras), seguido de resfriamento com imersão em água. Após a imersão em água os compósitos foram moídos em moinho granulador (RONE), disponível na Divisão de Materiais (AMR) do ComandoGeral de Tecnologia Aeroespacial (CTA).

Os compósitos moídos, previamente secos, foram injetados em molde contendo cavidades com dimensões específicas para ensaios mecânicos, utilizando uma Injetora Jasot 300/130, disponível na Divisão de Materiais (AMR) do Comando-Geral de Tecnologia Aeroespacial (CTA). Os compósitos obtidos com e sem o uso do PE-g-MAH como agente compatibilizante estão relacionados na Tabela 1. Esse estudo foi realizado para verificar a influência do PE-g-MAH como agente compatibilizante nas propriedades mecânicas dos compósitos.

Tabela 1 - Descrição dos compósitos utilizando agente compatibilizante

\begin{tabular}{|c|c|c|c|c|}
\hline Amostra & Fibra reforçada & $\begin{array}{c}\text { Quantidade de PEBD } \\
(\% \mathrm{~m} / \mathrm{m})\end{array}$ & $\begin{array}{c}\text { Quantidade de } \\
\text { reforço }(\% \mathrm{~m} / \mathrm{m})\end{array}$ & $\begin{array}{c}\text { Quantidade de } \\
\text { PE-g-MAH (phr) }\end{array}$ \\
\hline $\mathrm{CSC} 5 \%$ & Fibra de coco & 95 & 5 & 0 \\
\hline СCC $5 \%$ & Fibra de coco & 95 & 5 & 5 \\
\hline
\end{tabular}

\subsection{Ensaios Mecânicos}

Os ensaios de tração dos compósitos com e sem agente compatibilizante e do polietileno de baixa densidade puro foram realizados no Laboratório de Ensaios Mecânicos do Centro Universitário de Volta Redonda - UniFOA, em um equipamento da marca EMIC modelo DL10.000 a uma velocidade de $5 \mathrm{~mm} \cdot \mathrm{min}^{-1}$, com dimensões de acordo com a norma ASTM D 638 - 03 com $13 \mathrm{~mm}$ de largura, $165 \mathrm{~mm}$ de comprimento e $3 \mathrm{~mm}$ de espessura.

\subsection{Microscopia Eletrônica de Varredura}

A morfologia e a microestrutura das fibras de coco e dos compósitos foram analisadas por microscopia eletrônica de varredura (MEV) para avaliar a superfície das fibras e a fratura dos compósitos.
As amostras das fibras de coco verde e a superfície dos compósitos fraturados foram analisadas em um microscópio eletrônico de varredura JEOL JSM5310, disponível no Instituto Nacional de Pesquisas Espaciais/INPE em São José dos Campos, operando de 15 a 20 kW e utilizando detector de elétrons secundários. As amostras foram fixadas em um suporte, com auxílio de uma fita de carbono autocolante dupla face e submetidas ao recobrimento metálico com ouro.

\section{Resultados E Discussão}

As micrografias obtidas por MEV permitiram avaliar os aspectos morfológicos das fibras. A Figura 1 evidencia as micrografias das fibras de coco in natura obtidas em diferentes ampliações. 


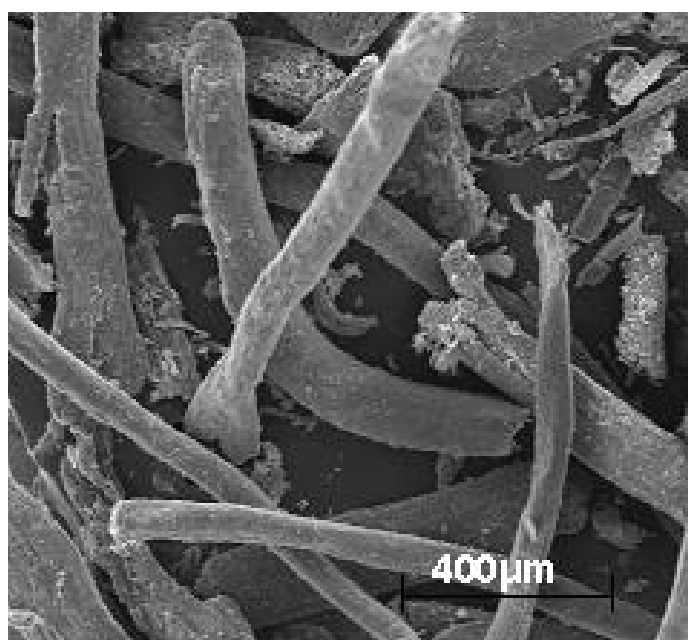

(a)

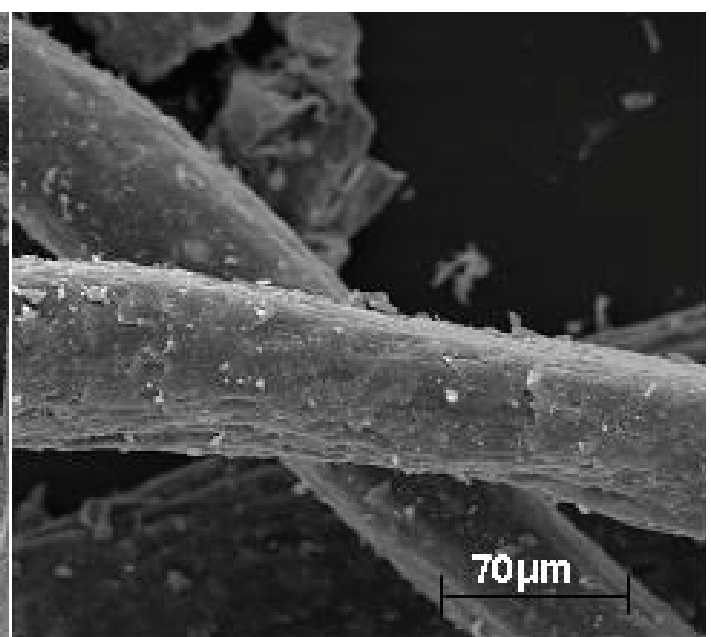

(b)

Figura 1 - Micrografias das fibras de coco in natura: (a) 100X; (b) 500X;

Analisando-se as micrografias das fibras de coco in natura observou-se uma superfície lisa, e a presença de extrativos cerosos envolvendo toda a superfície da fibra. As ceras e graxas que envolvem as fibras naturais impermeabilizam a celulose diminuindo a área de adesão entre a matriz e o reforço, sua remoção é de fundamental importância para o aumento das propriedades mecânicas dos compósitos ou o uso do agente compatibilizante poderá melhorar essas propriedades. A Figura 2 mostra um esquema da interação entre a superfície das fibras de celulose e o PE-g-MAH.

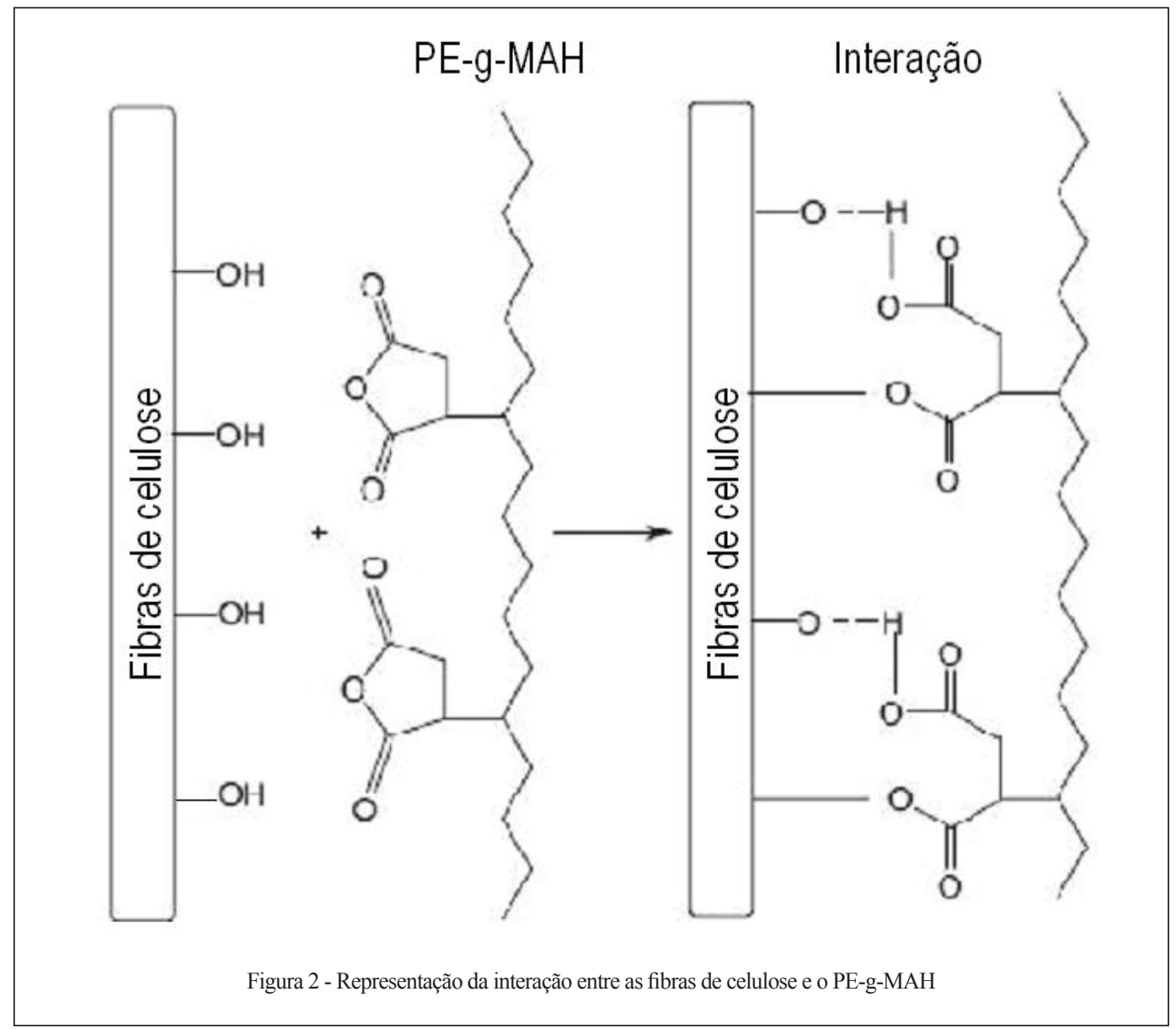

O tempo de mistura das fibras de coco in natura e da matriz com ou sem o uso do agente compatibilizante (PE-g-MAH) no homogenei- zador de plásticos não ultrapassou $85 \mathrm{~s}$. O uso do PE-g-MAH como agente compatibilizante nos compósitos influenciaram nas propriedades 
mecânicas dos compósitos quando comparados aos compósitos processados sem o uso do agente compatibilizante.

A Tabela 2 mostra os valores do limite de resistência à tração e seus respectivos alongamento e módulo.

Analisando-se os dados da Tabela 2 observou-se que a resistência à tração dos compósi- tos PEBD reforçados com fibras de coco compatibilizados (CCC5\%) e não compatibilizados (CSC5\%) aumentou, quando comparada ao PEBD puro. No entanto, os compósitos compatibilizados apresentaram melhores resistências à tração. Além disso, os compósitos compatibilizados apresentaram maior rigidez, quando comparados aos compósitos não compatibilizados.

Tabela 2 - Valores do limite de resistência à tração dos compósitos

\begin{tabular}{|c|c|c|c|}
\hline \multirow{2}{*}{ Amostras } & \multicolumn{2}{|c|}{ Propriedades } \\
\cline { 2 - 4 } & $\begin{array}{c}\text { Alongamento no limite de } \\
\text { resistência à tração (\%) }\end{array}$ & $\begin{array}{c}\text { Limite de resistência à } \\
\text { tração (MPa) }\end{array}$ & $\begin{array}{c}\text { Módulo de Elasticidade } \\
\text { (MPa) }\end{array}$ \\
\hline PEBD & $5,6 \pm 0,8$ & $12,2 \pm 1,1$ & $655,4 \pm 91,6$ \\
\hline CSC5\% & $4,4 \pm 0,3$ & $13,4 \pm 0,7$ & $742,3 \pm 69,5$ \\
\hline CCC5\% & $3,6 \pm 0,1$ & $17,1 \pm 0,2$ & $955,3 \pm 51,2$ \\
\hline
\end{tabular}

Essa diferença da rigidez nos compósitos ocorreu, pois o compatibilizante em contato com a superfície do reforço interagiu fortemente com as fibras através das ligações covalentes ou ligações de hidrogênio causando uma melhor interação entre fibra e matriz.
No entanto, a inserção de fibras na matriz de PEBD sem o uso do agente compatibilizante também influenciou na rigidez, quando comparado ao PEBD puro. A Figura 3 evidencia os módulos de elasticidade obtidos nos compósitos compatibilizados e não compatibilizados.

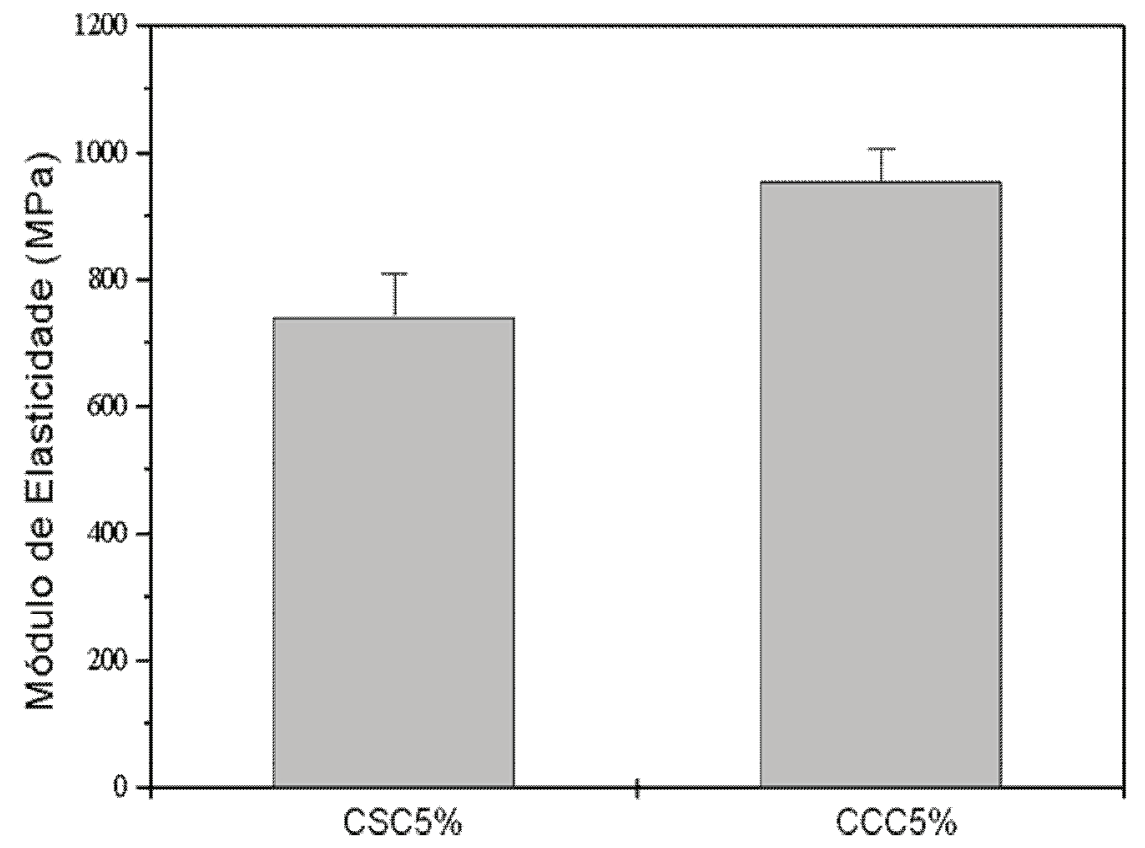

Figura 3 - Módulo de elasticidade em tração dos compósitos PEBD reforçados com fibras de coco com e sem agente compatibilizante.

O alongamento na tensão máxima dos compósitos diminui comparado ao PEBD puro. Comparando-se os compósitos notou-se que o uso do agente compatibilizante influenciou no alongamento na tensão máxima, devido à interação entre fibra/matriz, a qual contribui para o aumento da resistência. 
quais mostraram que os compósitos apresentaram fluxo da matriz, ou seja, deformação plástica do polietileno de baixa densidade. Comparando-se as micrografias, observou-se que os compósitos PEBD reforçados com fibras de coco compatibilizados apresentaram

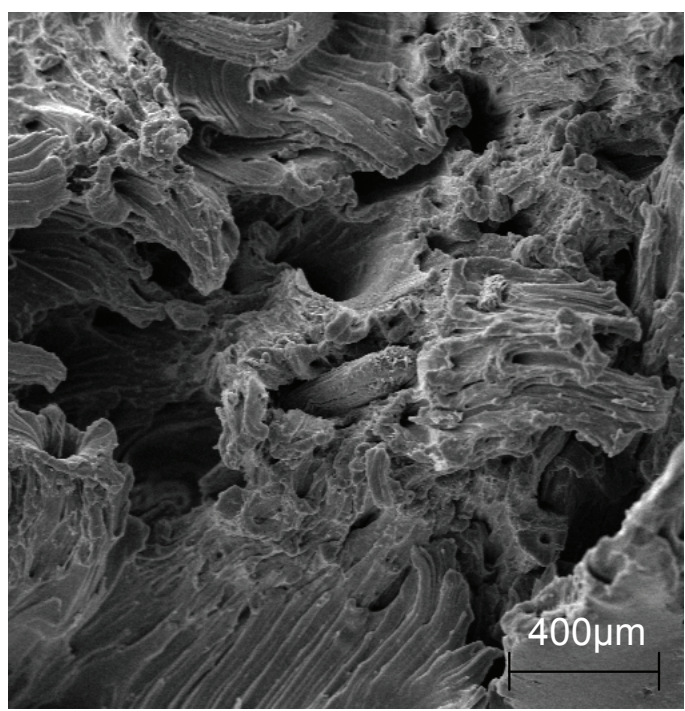

(a) melhor adesão entre fibra/matriz, quando comparadas aos compósitos PEBD reforçados com fibras de coco não compatibilizados. Além disso, foi observado que os compósitos não compatibilizados apresentaram pull out e arrancamento de fibras.

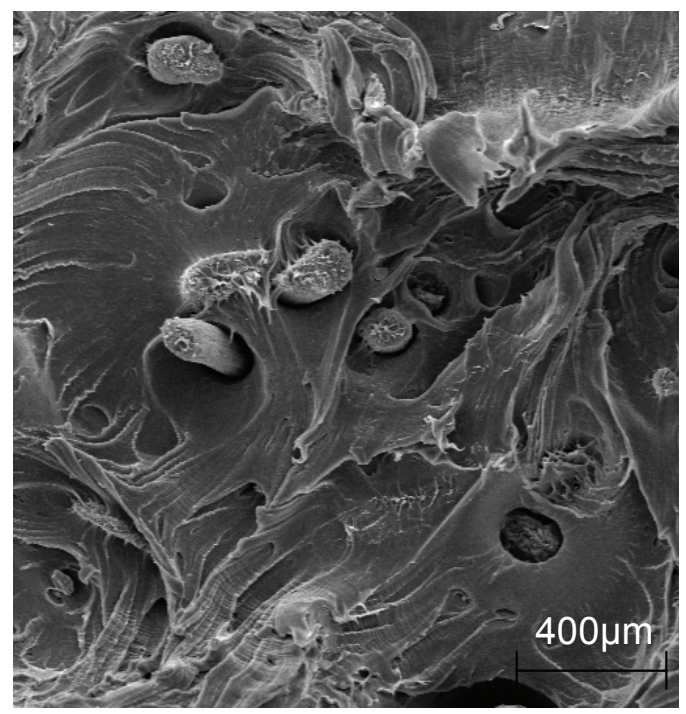

(b)

Figura 4 - Compósitos PEBD reforçados com fibras de coco: (a) sem agente compatibilizante; (b) com agente compatibilizante.

Dessa forma, pode-se afirmar que a adição de agente compatibilizante nos compósitos influenciou nas propriedades mecânicas, resultando em um material de diferentes propriedades dos componentes puros, constituindo uma opção para a redução de custos em aplicações industriais e também ajudando na preservação do meio ambiente.

\section{Conclusões}

O uso do agente compatibilizante influenciou nas propriedades mecânicas dos compósitos quando comparado aos compósitos não compatibilizados, pois o compatibilizante em contato com a superfície do reforço interagiu fortemente através das ligações de hidrogênio, causando uma melhor interação entre fibra e matriz. A técnica de microscopia eletrônica de varredura evidenciou esse resultado. No entanto, os compósitos não compatibilizados com PE-g-MAH apresentaram melhores propriedades mecânicas quando comparados ao PEBD puro.

\section{Referências Bibliográficas}

1. MANZINI, E.; VEZZOLI, C. O Desenvolvimento de Produtos Sustentáveis. 2 ed. São Paulo: Editora da Universidade de São Paulo, 2008, p.366.

2. GUIMARÃES, J. L.; FROLLINI E.; DA SILVA, C. G.; WYPYCH, F.; SATYANARAYANA,K.G.Characterization of banana, sugarcane bagasse and sponge gourd fibers of Brazil. Industrial Crops Production, v.30, p.407-415, 2009.

3. ZAH, R.; HISCHIER, R.; LEÃO, A. L.; BRAUN, I. Curauá fibers in the automobile industry - a sustainability assessment. Journal of Clear Production, v.15, p.1032-1040, 2007.

4. XU, X.; JAYARAMAN, K.; MORIN, C.; PECQUEUX, N. Life cycle assessment of wood-fiber-reinforced polypropylene composites. Journal of Materials Processing Technology, v.198, p.168-177, 2008.

5. LI, Y.; HU, C.; YU, Y. Interfacial studies of sisal fiber reinforced high density polyethylene (HDPE) composites. Composites: Part A, v.39, p.570-579, 2008. 
6. BECKERMANN, G. W.; PICKERING, K. L. Engineering and evaluation of hemp fibre reinforced polypropylene composites: Fibre treatment and matrix modification. Composites Part A, v.39, p.979-988, 2009.

7. BERTOTI, A. R.; LUPORINI, S.; ESPERIDIÃO, M. C. A. Effects of acetylation in vapor phase and mercerization on the properties of sugarcane fibers. Carbohydrate Polymers, v.77, p.20-24, 2009.

8. GU, H. Tensile behaviours of the coir fiber and related composites after $\mathrm{NaOH}$ treatment. Materials \& Desing, v.30, p.3931-3934, 2009.

9. JOHN, M. J.; ANANDJIWAL, R. D. Chemical modification of flax reinforced polypropylene composites. Composites: Part A, v.40, p.442-448, 2009.

10. MULINARI, D. R.; VOORWALD, H. J. C.; CIOFFI, M. O. H.; DA SILVA, M. L. C. P.; ROCHA, G. J. M. Surface modification of sugarcane bagasse cellulose and its effect on mechanical and absorption properties of sugarcane bagasse cellulose/HDPE composites. Bioresource, v.5, p.661-671, 2010.

11. MULINARI, D. R.; VOORWALD, H. J. C.; CIOFFI, M. O. H.; DA SILVA, M. L. C. P.; LUZ, S. M. Preparation and properties of HDPE/sugarcane bagasse cellulose composites obtained for thermokinetic mixer. Carbohydrate Polymers, v.75, p.317-320, 2009a.

12. MULINARI, D. R.; VOORWALD, H. J. C.; CIOFFI, M. O. H.; DA SILVA, M. L. C. P.; LUZ, S. M. Preparation and properties of HDPE/sugarcane bagasse cellulose composites obtained for thermokinetic mixer. Carbohydrate Polymers, v.75, p.317-320, 2009b.

13. 13. ARAÚJO, J. R.; WALDMAN, W. R.; DE PAOLI, M. A. Thermal properties of high density polyethylene composites with natural fibres: Coupling agent effect. Polymer Degradation and Stability, v.93, p.1170-1175, 2008.

Endereço para Correspondência:

Daniella Regina Mulinari

daniella.mulinari@foa.org.br

Departamento de Engenharia - UniFOA

Centro Universitário de Volta Redonda

Campus Três Poços

Av. Paulo Erlei Alves Abrantes, $n^{\circ}$ 1325,

Três Poços - Volta Redonda / RJ

CEP: 27240-560

Informações bibliográficas:

Conforme a NBR 6023:2002 da Associação Brasileira de Normas Técnicas (ABNT), este texto científico publicado em periódico eletrônico deve ser citado da seguinte forma: Conforme a NBR 6023:2002 da Associação Brasileira de Normas Técnicas (ABNT), este texto científico publicado em periódico eletrônico deve ser citado da seguinte forma:
OLIVEIRA, Taysa Ávila; TEIXEIRA, Alison; MULINARI, Daniella Regina; GOULART, Shane Aparecida Soares. Avaliação do uso de agente compatibilizante no comportamento mecânico dos compósitos Pebd reforçados com Fibras de Coco Verde. Cadernos UniFOA. Volta Redonda, Ano V, n. 14, dezembro 2010. Disponível em: $<$ http://www.unifoa.edu.br/cadernos/edicao/14/11.pdf $>$ 